\title{
Utility of transient elastography in evaluation and follow-up of Budd-Chiari syndrome patients after endovascular intervention
}

Hend Elsaied Ebada ${ }^{* *} \mathbb{D}$, Mohamed Fawzy Montasser', Eman Fathy Barakat', Sara Mahmoud Abdelhakam', Mohamed Shaker Ghazy² and Ahmed Talaat Elganzory ${ }^{1}$

\begin{abstract}
Background: Liver stiffness measurement (LSM) using transient elastography (TE) is a common noninvasive method for assessing hepatic fibrosis. The purpose of this study was to show the utility of LSM in the evaluation and short-term follow-up of Budd-Chiarri syndrome (BCS) patients as well as to investigate the relationships between LSM, hepatic venous pressure, and liver fibrosis. We conducted a prospective cohort study in which 25 patients with a confirmed diagnosis of primary BCS underwent endovascular intervention for BCS treatment. All patients had pressure gradient changes across the stenosed hepatic veins/inferior vena cava (HV/IVC) measured before and after the procedure, a transvenous liver biopsy during the procedure, and LSM (using Transient Elastography 3 days before and 3 months after the intervention as a short-term follow-up). Comparisons and correlations were made between liver stiffness, hepatic venous pressure, and fibrosis.

Results: The HV/IVC pressure gradient measured immediately before and after the intervention had a direct proportionate correlation with the LS measured 3 days before and 3 months after the intervention $(r=0.41 \& 0.44$, p: $0.04 \& 0.03$, respectively). LSM drops significantly 3 months after intervention (from $51.62 \pm 22 \mathrm{kPa}$ to $28.80 \pm$ $19.25 \mathrm{kPa}$ ( $p: 0.001)$ ). There is a significant improvement in LSM post-intervention among patients with moderate to severe fibrosis (METAVIR scores of $\geq$ F2 before intervention ( $p$ : 0.001).

Conclusions: LSM in BCS patients reflects congestion rather than fibrosis stage. TE can be used for the short-term monitoring of patients with BCS following the endovascular intervention as a noninvasive tool.
\end{abstract}

Keywords: Hepatic fibrosis, Hepatic venous pressure gradient, Liver congestion, Liver stiffness

\footnotetext{
*Correspondence: dr.hend@med.asu.edu.eg; drhannod21@gmail.com

'Departement of Tropical Medicine, Ain Shams University, Cairo, Abbasia

11341, Egypt

Full list of author information is available at the end of the article
}

\section{Springer Open}

(c) The Author(s). 2021 Open Access This article is licensed under a Creative Commons Attribution 4.0 International License, which permits use, sharing, adaptation, distribution and reproduction in any medium or format, as long as you give appropriate credit to the original author(s) and the source, provide a link to the Creative Commons licence, and indicate if changes were made. The images or other third party material in this article are included in the article's Creative Commons licence, unless indicated otherwise in a credit line to the material. If material is not included in the article's Creative Commons licence and your intended use is not permitted by statutory regulation or exceeds the permitted use, you will need to obtain permission directly from the copyright holder. To view a copy of this licence, visit http://creativecommons.org/licenses/by/4.0/. 


\section{Background}

In BCS, hepatic venous outflow obstruction causes venous stasis, post-sinusoidal portal hypertension, and a decrease in portal perfusion, culminating in ischemic damage to hepatocytes and hepatic cell inflammation. These biological factors all contribute to increased liver stiffness, which can be measured using one-dimensional transient elastography (TE) [1].

Liver stiffness (LS) is a noninvasive method for estimating liver fibrosis. Although previously thought to be inappropriate for BCS due to liver congestion, LS is now thought to be capable of monitoring treatment outcomes in BCS after balloon angioplasty, albeit without correlating with liver fibrosis [2].

LSM correlates with the hepatic venous pressure gradient (HVPG) [3, 4], the gold standard of portal hypertension (PH) evaluation [5], and thus could be used to indirectly assess $\mathrm{PH}$ in patients with advanced chronic liver disease (ACLD) [6].

According to the most recent Baveno Consensus on $\mathrm{PH}$, LSM values greater than $20-25 \mathrm{kPa}$ can noninvasively rule in clinically significant $\mathrm{PH}(\mathrm{CSPH})$ in ACLD patients [3].

LSM, on the other hand, does not only reflect liver fibrosis but also many other conditions [7-10], including liver congestion [9]. As a result, LSM could play a role in the evaluation of BCS because the hepatic venous flow obstruction found in these patients typically leads to liver congestion and a post-sinusoidal form of $\mathrm{PH}$.

The goal of treatment (via endovascular intervention) in patients with BCS is to relieve hepatic congestion, thereby improving hepatocyte function and allowing portal hypertension to resolve, and TE can measure this indirectly $[11,12]$.

The current study aimed to demonstrate the utility of TE in the diagnosis, staging, and follow-up of liver fibrosis in patients with BCS before and after the endovascular intervention. In addition, TE was used to determine if there is a correlation between the preintervention fibrosis stage (using Meta-analysis of Histological Data in Viral Hepatitis (METAVIR)) and posttreatment LSM.

Only a few studies, mostly case series, have looked at the role of TE in the diagnosis of liver fibrosis in BCS patients, as well as the possibility of using TE as a noninvasive tool to monitor these patients after endovascular intervention.

\section{Methods}

\section{Patients}

This prospective cohort study enrolled 25 Egyptian patients who had primary BCS confirmed by Doppler ultrasound (described in the following section) performed by a single radiologist from our group. Before participating, all patients provided written informed consent, and the local
Research Ethical Committee approved the study protocol, in accordance with the ethical guidelines of the 1975 Declaration of Helsinki.

\section{Exclusion criteria}

Patients with other etiologies of liver disease unrelated to BCS (e.g., viral, autoimmune, or metabolic), those who had BCS due to secondary causes (e.g., tumoral hepatic venous (HV) or inferior vena caval (IVC) invasion or compression), patients who had a contraindication for transjugular intrahepatic portosystemic shunt (TIPS) (such as severe congestive heart failure, uncontrolled sepsis, and advanced liver disease), and patients who had one or more confounder which could affect the proper assessment of liver stiffness (LS) (e.g., BMI > 30, acute hepatitis, and extrahepatic cholestasis) were excluded.

\section{Tools of the study}

Complete histories and clinical examinations were recorded for all included patients, and laboratory investigations included a complete blood count (CBC), a liver profile, and a coagulation profile. As part of our protocol, we performed a thorough thrombophilia workup for all patients with a confirmed diagnosis of primary BCS to determine the underlying etiology (s).

Abdominal Doppler ultrasonography was performed to assess: liver and splenic size, ascites status, caudate lobe enlargement, mottled parenchyma, presence of hepatic focal lesions, the status of the major HVs whether patent or occluded (long or short segment occlusion), and the number of occluded veins; the status of IVC, pattern of vascular occlusion (i.e., hepatic vein occlusion (HVO) and/or IVC occlusion (IVCO)), portal vein (PV) and intra \& extrahepatic collaterals. In cases of nonconclusive Doppler ultrasound findings or the presence of hepatic focal lesions, data from multi-slice computed tomography (CT) with venography and portography and/or magnetic resonance venography (MRV) and dynamic MRI were recorded to clarify their nature. Following that, all patients underwent upper endoscopy to determine the status of gastroesophageal varices and portal hypertensive gastropathy and to treat them accordingly before beginning the anticoagulation therapy.

\section{Endovascular intervention and pressure gradient measurement}

The intervention was chosen based on the location and extent of the venous occlusion. TIPS was performed on 18 patients, with 7 undergoing angioplasty in the form of IVC balloon dilatation without stenting (5 patients) and hepatic vein stenting (2 patients). Pressure gradients were measured both before and after the procedure. Pressure gradients were measured using $4 \mathrm{~F}$ or $5 \mathrm{~F}$ 
multipurpose catheters across the stenosed HV segment between the patent distal HV segment and the IVC or right atrium in patients undergoing $\mathrm{HV}$ angioplasty and/ or stent implantation. Gradients were measured in patients with IVC occlusion or stenosis across the patent segment of the IVC and right atrium. Portosystemic gradients were measured after TIPS creation.

\section{LS measurement technique}

All included patients had their liver stiffness assessed using a Fibroscan Echosens 502 (M probe and XL probe) by a single well-trained operator. For all patients, the LSM was taken twice, 3 days before the endovascular intervention and 3 months later as a short-term followup. Patients who had significant large ascites before the examination underwent paracentesis. Patients were placed on their backs in the dorsal decubitus position, with their right arm in full abduction. The right hepatic lobe was used for LS measurements. In a right intercostal space, a $3.5-\mathrm{MHz}$ US transducer mounted on the axis of a vibrator was placed. As for elastic shear waves, a 50$\mathrm{Hz}$ vibration with an amplitude of $2 \mathrm{~mm}$ and a pulse tracking frequency of $3.5 \mathrm{MHz}$ was propagated into the liver. The mean LS value was calculated in kilopascals for each patient who had at least ten successful measurements. The range of LSM values is $0-75.4 \mathrm{kPa}$. The approximate range of LSM values from F0 to F4 corresponds to different stages of fibrosis:

- F0: $0-4.9 \mathrm{kPa}$

- F1: $5-7.4 \mathrm{kPa}$

- F2: $7.5-9.9 \mathrm{kPa}$

- F3: $10-14.9 \mathrm{kPa}$

- F4: $15-75 \mathrm{kPa}$

\section{Liver biopsy}

All patients had a percutaneous transjugular liver biopsy once during the interventional procedure. The METAVIR scoring system was used to assess the fibrosis stage [13]. The patients were divided into two groups: those with a fibrosis score $<$ F2 and those with a fibrosis score $\geq$ F2. The differences in changes in LS measurements after interventional procedures between these groups were analyzed.

\section{Statistical techniques}

A biomedical statistician carried this study's statistical review out. Data was gathered, revised, coded, and entered using the Statistical Package for Social Science (SPSS) version 23.

The qualitative data were presented as numbers and percentages, whereas the quantitative data were presented in the form of mean, standard deviations (SD), and ranges. Multiple comparisons between groups were carried out. LSD (post hoc range test) was used to make multiple comparisons between pairs of groups, with the findings displayed as mean and SD. To compare clinical data before and after the intervention, the MacNemar test was performed. The statistical significance of the variations in LSM values before and after the endovascular operation was determined using a paired samples $t$ test. Pearson's correlation coefficient was used to analyze the correlations between changes in pressure gradients across stenosed HVs/IVC and LS measures after interventional operations. The relationship between LSM before and after the intervention and METAVIR score was determined using a marginal homogeneity test. SPSS version 17 was used to analyze all of the data. A $p$ value of less than 0.05 was deemed significant.

\section{Results}

In this study, 25 Egyptian patients with primary BCS were included. There were 13 males and 12 females. The average age was $36.24 \pm 9.34$ years (range: $19-55$ years). In $80 \%$ of patients with BCS, the chronic presentation was the most common, with painful hepatomegaly and increasing abdominal enlargement leading to ascites being the most common symptoms. TIPS was performed on 18 patients, whereas 7 underwent angioplasty \pm stenting in the form of IVC balloon dilatation (5 patients) and hepatic vein stenting (2 patients). One of the patients became pregnant shortly after the intervention and was lost to follow-up; LSM was not used on her.

There was a total clearance of ascites in 18 patients after the endovascular intervention, and only grade 1 ascites in 6 patients. In addition, liver functions improved significantly. Table 1 compares the clinical, laboratory, and radiological characteristics of patients before and 3 months after the intervention. Table 1 shows that following the intervention, the HV/IVC pressure gradient decreased markedly in all patients $(p=0.001)$.

The LSM measured at 3-month follow-up following the intervention showed significant improvement $(p<0.001)$ (Table 1 \& Fig. 1).

\section{Correlation between HV/IVC pressure gradient \& LSM}

Before the intervention, the mean SD changes in HV/ IVC pressure gradient in all patients were $23.8 \pm 55$ $\mathrm{mmHg}$, and the mean SD changes in LSM in these patients were $51.62 \pm 22 \mathrm{kPa}$, while the mean changes in HV/IVC pressure gradient were $8.00 \pm 2 \mathrm{mmHg}$ and in LSM were $28.80 \pm 19.25 \mathrm{kPa}$ at 3 months (Table 1 ).

The correlation coefficients between changes in pressure gradients and changes in LSM before and after the intervention were 0.41 and 0.44 , indicating a moderate positive association between these two nonparametric measures (Fig. 2a \& b). 
Table 1 Characteristics of patients before and after endovascular intervention

\begin{tabular}{|c|c|c|c|}
\hline Characterstics of patients $^{\mathrm{a}}(n=24)$ & Pre-intervention & Post-intervention & $p$ value \\
\hline \multicolumn{4}{|l|}{ Clinical } \\
\hline Jaundice & $4(16 \%)$ & 0 & 0.13 \\
\hline Encephalopathy & $2(8 \%)$ & 0 & 0.5 \\
\hline LL edema & $14(56 \%)$ & 0 & $<0.001$ \\
\hline Abdominal enlargement & $14(56 \%)$ & 0 & $<0.001$ \\
\hline Tender liver & $17(68 \%)$ & $5(20.8 \%)$ & 0.003 \\
\hline \multicolumn{4}{|l|}{ Laboratory } \\
\hline Bilirubin & $1.16 \pm 0.49$ & $0.97 \pm 0.21$ & 0.02 \\
\hline ALT & $33.12 \pm 8.71$ & $22.96 \pm 7.18$ & $<0.001$ \\
\hline AST & $25.80 \pm 8.3$ & $17.46 \pm 6.268$ & $<0.001$ \\
\hline \multicolumn{4}{|l|}{ Radiological (Duplex ultrasound) } \\
\hline \multirow[t]{3}{*}{ Ascites } & Grade 1: 11 & No ascites 18 & $<0.001$ \\
\hline & Grade 2: 9 & Grade 1: 6 & \\
\hline & Grade 3: 4 & & \\
\hline Hepatomegaly & $18(72 \%)$ & $18(72 \%)$ & 1 \\
\hline Intrahepatic collaterals & $7(28 \%)$ & 0 & $<0.001$ \\
\hline HVPG & $\begin{array}{l}23.80 \pm 6.55 \\
(15-40)\end{array}$ & $\begin{array}{l}8.00 \pm 2 \\
(4-12)\end{array}$ & $<0.001$ \\
\hline LSM mean (range) & $\begin{array}{l}51.62 \pm 22 \\
(11.4-75)\end{array}$ & $28.80 \pm 19.25(8.7-75)$ & $<0.001$ \\
\hline Liver biopsy (METAVIR staging) & $\begin{array}{l}\text { F1:7 (28\%) } \\
\text { F2: } 6(24 \%) \\
\text { F3: 3(12\%) } \\
\text { F4: } 9(36 \%)\end{array}$ & & \\
\hline
\end{tabular}

${ }^{a}$ Clinical, laboratory, and radiological characteristics (Duplex US) of the patents were assessed once before and again 3 months post-intervention. HVPG was measured immediately before and after intervention. Liver biopsy was done only during the procedure using transvenous approach (i.e., before the endovascular intervention)

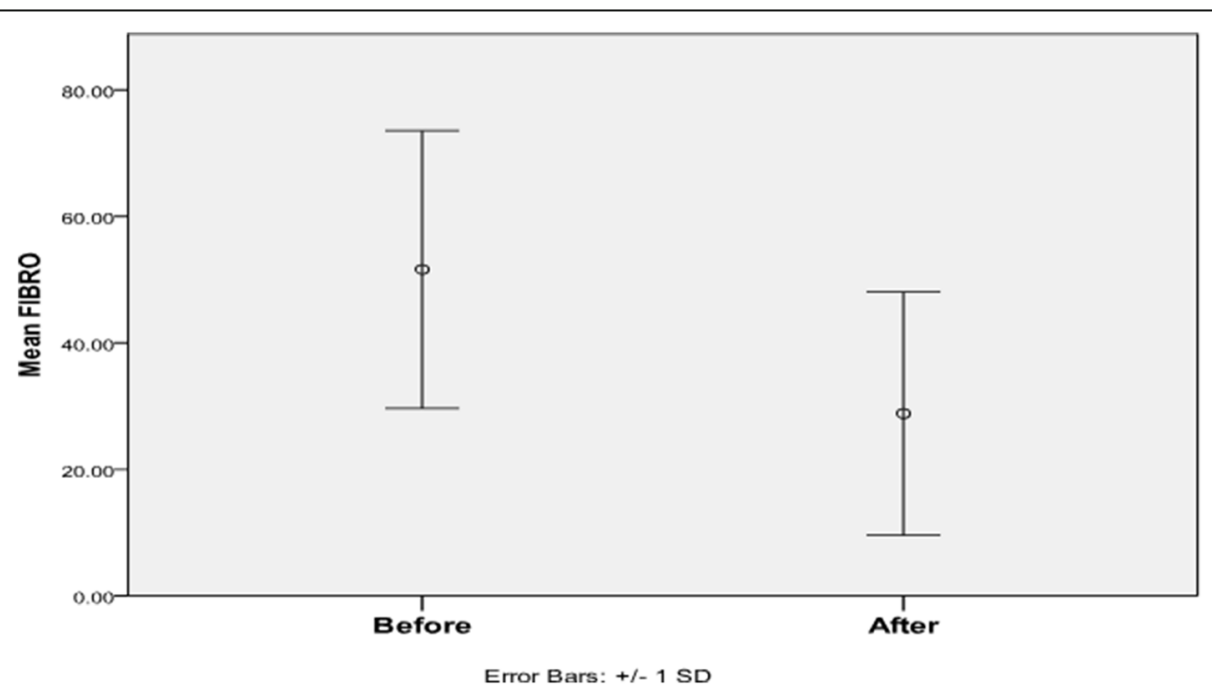

Fig. 1 Liver stiffness measurement pre- and post-intervention 

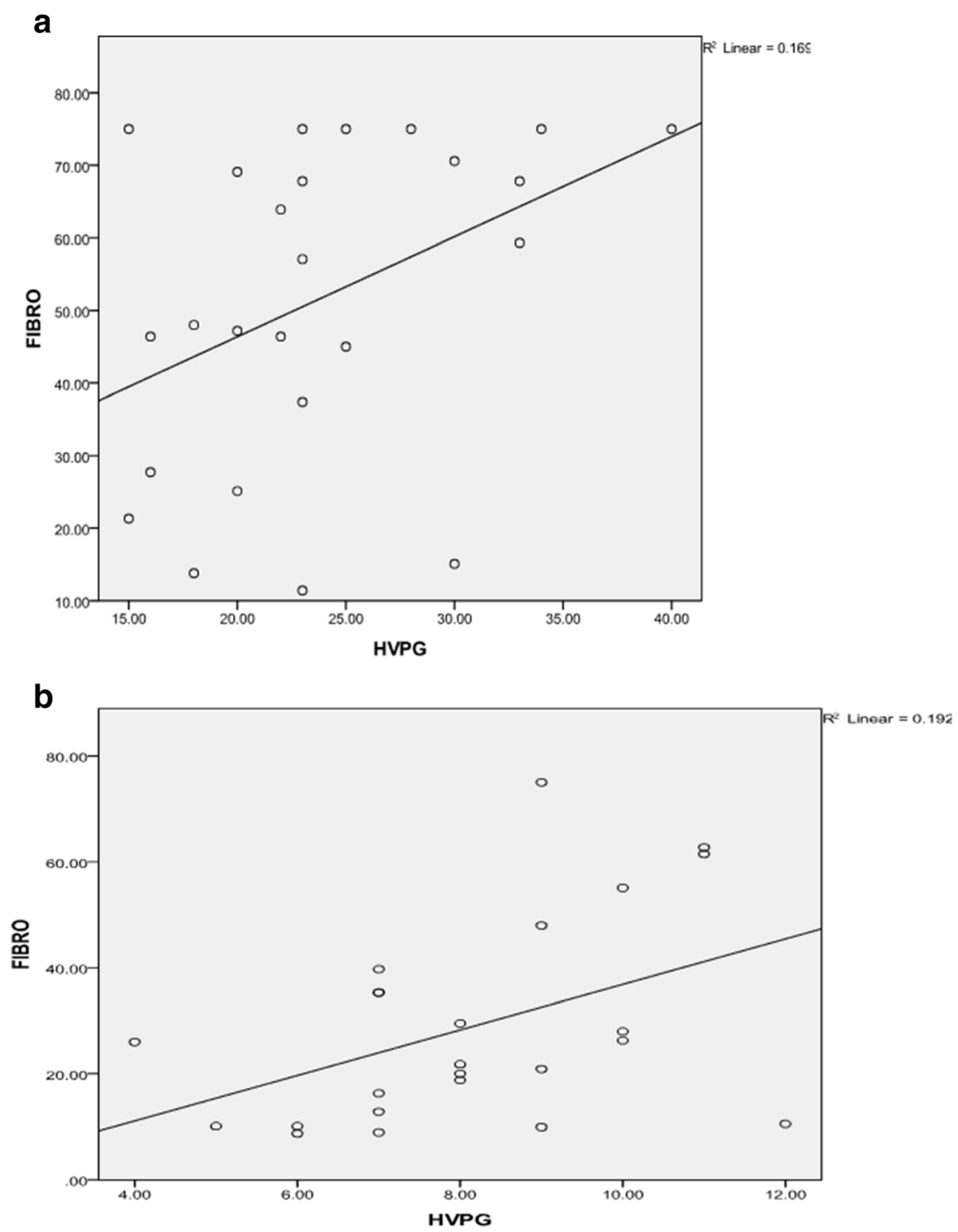

Fig. 2 a Correlation between LSM and HV/IVC pressure gradient before intervention. b Correlation between LSM and HV/IVC pressure gradient at 3-month follow-up post-intervention

\section{Correlation between LSM and METAVIR staging}

When we used METAVIR staging to try to find a link between changes in LSM and histological fibrosis staging, we discovered a significant difference between the METAVIR score of the liver biopsy and the LSM, whether performed 3 days before the intervention or 3 months after the intervention $(p=0.001)$. However, the difference between the results of LSM post-intervention \& liver biopsy staging became less compared with the difference before intervention (Table 2).

We found no significant improvement in LSM postintervention in the group of patients with METAVIR scores of $<\mathrm{F} 2(n=6)$, while there was a substantial improvement in LSM post-intervention in the group of patients whose METAVIR scores were $\geq$ F2 $(n=18)$ (Table 3).

\section{Discussion}

The goal of this study was to demonstrate how useful TE was for diagnosing, staging, and monitoring liver fibrosis in patients with BCS before and after endovascular intervention, also to identify if there is a relation between LSM and the pre-intervention fibrosis stage (METAVIR). 
Table 2 Relation between pre- \& post-intervention LSM and liver biopsy (METAVIR score)

\begin{tabular}{|c|c|c|c|c|c|c|c|}
\hline \multirow[t]{2}{*}{ LSM } & & \multicolumn{5}{|c|}{ Liver biopsy (METAVIR staging) } & \multirow[t]{2}{*}{$p$ value } \\
\hline & & $\mathrm{F} 1$ & F2 & F3 & F4 & Total & \\
\hline \multirow[t]{3}{*}{ LSM 3 days pre-intervention } & F3 & 2 & 0 & 0 & 0 & 2 & \multirow[t]{3}{*}{$<0.001$} \\
\hline & F4 & 5 & 6 & 3 & 9 & 23 & \\
\hline & Total & 7 & 6 & 3 & 9 & 25 & \\
\hline \multirow[t]{4}{*}{ LSM 3 months post-intervention } & F2 & 1 & 0 & 0 & 0 & 1 & \multirow[t]{4}{*}{0.001} \\
\hline & F3 & 2 & 2 & 1 & 1 & 6 & \\
\hline & $\mathrm{F} 4$ & 3 & 4 & 2 & 8 & 17 & \\
\hline & Total & 6 & 6 & 3 & 9 & 24 & \\
\hline
\end{tabular}

LSM Liver stiffness measurement

All patients who underwent HV angioplasty/stenting or TIPS in the current study had a significant reduction in their HV/IVC pressure gradient following the intervention, resulting in an immediate reduction in hepatic congestion ( $p$ value $=0.001$ ). This is supported by two recent studies involving 19 and 32 BCS patients, respectively. In these investigations, the pressure gradient drops significantly after the intervention $[12,14]$.

The pre-procedure LSM mean $(51.62 \pm 22 \mathrm{kPa}$, encompassing 23 patients in the F4 stage and 2 patients in the F3 range) in our data was higher than the cut-off value of $10.4 \mathrm{kPa}$ (above which is widely believed to characterize F4 fibrosis (liver cirrhosis)) [15, 16]. Our data also demonstrated a considerable improvement in hepatic fibrosis after reducing liver congestion, going from $51.62 \pm 22 \mathrm{kPa}$ pre-intervention down to $28.8 \pm$ $19.25 \mathrm{kPa} 3$ months afterward. This information could drive clinicians to request more imaging studies to confirm BCS diagnosis. Dajti et al. stated that such high LSM values are uncommon in other liver illnesses, such as advanced chronic liver disease (ACLD) or other disorders that cause hepatic congestion [17].

Pre- and post-intervention, LSM was found to be positively correlated with $\mathrm{HV} / \mathrm{IVC}$ pressure gradient $(r=$ $0.41, p=0.04)$ and $(r=0.44, p=0.03)$, respectively. As a result, TE follow-up monitoring can be used to assess the therapeutic benefits in BCS patients.

Wang et al. showed a direct association between HVPG and LSM before intervention (the coefficient of correlation between LSM and hepatic venous pressure before angioplasty was $r=0.701,(p 0.001))$ [12], which is consistent with our findings.

Mukund et al., in contrast to our findings, observed no relation between changes in LSM and pressure gradient [14].

There was a significant difference between the histological stage of fibrosis and the LSM performed 3 days before intervention $(p 0.001)$ and 3 months after intervention $(p=0.001)$ in the current study.

Our findings are consistent with those of Wang et al. [12], who found that LSM was related to hepatic venous pressure rather than the degree of fibrosis in BCS patients. They found that LSM did not correlate with the degree of fibrosis in histological specimens before angioplasty $(r=0.170, P=0.22)$. LSM still did not correlate with the degree of fibrosis 2 days after the intervention $(r=0.223, p=0.220)$, and there was no significant difference in LSM between different fibrosis stages before angioplasty [12]. These findings contradict prior findings that LSM is positively correlated with the severity of fibrosis $[1,14]$.

This is maybe because LSM is more affected by hepatic venous pressure (i.e., liver congestion due to post-sinusoidal portal hypertension in BCS patients leads to higher LSM).

Unlike Mukund et al., who found no significant difference in LS values post-intervention between both groups with METAVIR fibrosis scores of $\leq 2(n=7)$ and $>2(n=5), p=0.5714$, our findings showed no significant improvement in LSM post-intervention among the

Table 3 Comparison between pre- \& post-intervention LSM and different stages of METAVIR score $(n=24)$

\begin{tabular}{|c|c|c|c|c|c|c|}
\hline & Min. & Max. & Mean & SD & $t^{*}$ & $p$ value \\
\hline \multicolumn{7}{|c|}{ METAVIR < F2: $(n=6)$} \\
\hline LSM before & 11.40 & 75.00 & 39.34 & 26.98 & 2.32 & 0.07 \\
\hline LSM after & 8.70 & 39.70 & 18.77 & 12.31 & & \\
\hline \multicolumn{7}{|c|}{ METAVIR $\geq \mathbf{F 2 :}(n=18)$} \\
\hline LSM before & 15.10 & 75.00 & 56.39 & 18.45 & 4.37 & $<0.001$ \\
\hline LSM after & 9.90 & 75.00 & 32.14 & 20.24 & & \\
\hline
\end{tabular}


group of patients whose fibrosis stage was $<$ F2 $(n=6, p$ $=0.07)$. However, among patients with moderate-tosevere fibrosis, there is a significant improvement in LSM post-intervention (scores $\geq \mathrm{F} 2, n=18, p$ 0.001). The substantially varying outcomes of liver elasticity can explain this in relation to the METAVIR fibrosis score [18]. These findings supported the idea that hepatic congestion, rather than fibrosis score, is the primary determinant of liver elasticity in BCS patients [19].

Mancuso et al. recently claimed that LS alterations from baseline could predict BCS outcomes when treated with only medicinal therapy. They measured LS every 4-5 months with FibroScan Echosens, and after an average 10-month follow-up, three patients, two with improved LS and one with stable LS, had stable liver disease, whereas one with worsening LS developed refractory ascites, with an increase in both creatinine and bilirubin, and was referred for evaluation of both liver and kidney transplantation [20]. In two cases with BCS, a recent case report investigated the efficacy of the hepatic vein $(\mathrm{HV})$ waveform by Doppler ultrasonography and quantified liver stiffness (LS) using transient elastography (TE) before and after percutaneous transhepatic angioplasty (PTA). Serial monitoring of the HV waveform and the LS value, according to the authors, allows for the safe management of patients with BCS who may require PTA [21].

\section{Conclusions}

Our findings imply we could utilize LSM as a sensitive noninvasive method to monitor hemodynamic changes in BCS patients after therapy, and that we might use it for short-term follow-up of BCS patients following endovascular intervention.

\section{The limitations of the current study}

The relatively small number of the studied patients and the limited duration of follow-up are the limitations of the current study. Frequent liver biopsies after the endovascular intervention with fibrosis staging using the METAVIR staging and comparing their results to the results of repeated LSM at the same time for validation of the role of LSM in long-term follow-up of BCS patients are recommended.

\section{Recommendations}

We highly encouraged a multicenter study or larger cohort with longer follow-up duration and serial LSM after endovascular interventions to confirm the role of LSM in short- and long-term follow-up of BCS patients, its potential prognostic value, and stratifying severity of the disease.

\section{Abbreviations}

ACLD: Advanced chronic liver disease; BMI: Body mass index; CBC: Complete blood count; CT: Computed tomography; BCS: Budd-Chiari syndrome; HV: Hepatic vein; HVPG: Hepatic venous pressure gradient; HVO: Hepatic venous occlusion; IVC: Inferior vena cava; LS: Liver stiffness; LSM: Liver stiffness measurement; MRI: Magnetic resonance imaging; MRV: Magnetic resonance venography TE; PH: Portal hypertension; PTA: Percutaneous transhepatic angioplasty; TIPS: Transjugular intrahepatic portosystemic shunt; TE: Transient elastography

\section{Acknowledgements}

The authors gratefully acknowledge all members of the Budd-Chiari study group at Tropical Medicine Department, Ain Shams University.

\section{Authors' contributions}

All authors contributed to the study conception and design. Material preparation, data collection, and analysis were performed by MFM, EFB, MAS, AFE, and SMA. The first draft of the manuscript was written by HEE and MSG, and all authors commented on previous versions of the manuscript. The authors read and approved the final manuscript.

\section{Funding}

No funding was received from any funding agency.

Availability of data and materials

The datasets used and/or analyzed during the current study are available from the corresponding author on reasonable request.

\section{Declarations}

Ethics approval and consent to participate

The study was approved by the Research Ethical Committee of Faculty of Medicine, Ain Shams University according to the ethical guidelines of the 1975 Declaration of Helsinki. Written informed consents have been obtained from all included patients in this study (ethical approval number and date: $177 / 2017$ and its date was 20/6/2017).

Consent for publication

Consent for publication is obtained from the participants.

\section{Competing interests}

All authors declare that they have no competing interests.

\section{Author details}

${ }^{1}$ Departement of Tropical Medicine, Ain Shams University, Cairo, Abbasia 11341, Egypt. ${ }^{2}$ Department of Radiodiagnosis \& Interventional Radiology, Ain Shams University, Cairo, Abbasia 11341, Egypt.

Received: 27 May 2021 Accepted: 10 August 2021

Published online: 15 December 2021

\section{References}

1. Perelló A, García-Pagán JC, Gilabert R, Suárez Y, Moitinho E, Cervantes F, Reverter JC, Escorsell A, Bosch J, Rodés J (2002) TIPS is a useful long-term derivative therapy for patients with Budd-Chiari syndrome uncontrolled by medical therapy. Hepatology 35(1):132-139. https://doi.org/10.1053/jhep.2 002.30274

2. He F, Zhao H, Dai S, Wu Y, Wang L, Huang H, Yue Z, Fan Z, Dong X, Liu F (2016) Transjugular intrahepatic portosystemic shunt for Budd-Chiari syndrome with diffuse occlusion of hepatic veins. Sci Rep 6(1):36380. https://doi.org/10.1038/srep36380

3. (2015) EASL-ALEH Clinical Practice Guidelines: non-invasive tests for evaluation of liver disease severity and prognosis. Hepatol 63(1):237-264. https://doi.org/10.1016/j.jhep.2015.04.006

4. Song Z, Ma J, Huang C et al (2018) Comparison of three cutoffs to diagnose clinically significant portal hypertension by liver stiffness in chronic viral liver diseases: a meta-analysis. Eur Radiol 28(12):5221-5230. https://doi.org/10.1 007/s00330-018-5478-z

5. Bosch J, Abraldes JG, Berzigotti A, García-Pagan JC (2009) The clinical use of HVPG measurements in chronic liver disease. Nat Rev Gastroenterol Hepatol 6(10):573-582. https://doi.org/10.1038/nrgastro.2009.149 
6. Berzigotti A (2017) Non-invasive evaluation of portal hypertension using ultrasound elastography. Hepatol. 67(2):399-411. https://doi.org/10.1016/j. jhep.2017.02.003

7. Arena U, Vizzutti F, Corti G, Ambu S, Stasi C, Bresci S, Moscarella S, Boddi V Petrarca A, Laffi G, Marra F, Pinzani M (2008) Acute viral hepatitis increases liver stiffness values measured by transient elastography. Hepatology. 47(7): 380-384. https://doi.org/10.1002/hep.22007

8. Millonig FM, Reimann S, Friedrich C et al (2008) Extrahepatic cholestasis increases liver stiffness (FibroScan) irrespective of fibrosis. Hepatology. 48(5): 1718-1723. https://doi.org/10.1002/hep.22577

9. Colli A, Pozzoni P, Berzuini A, Gerosa A, Canovi C, Molteni EE, Barbarini M, Bonino F, Prati D (2010) Decompensated chronic heart failure: increased liver stiffness measured by means of transient elastography. Radiology. 257(3):872-878. https://doi.org/10.1148/radiol.10100013

10. Dietrich J, Bamber A, Berzigotti C et al (2017) EFSUMB guidelines and recommendations on the clinical use of liver ultrasound elastography, update 2017 (long version). Ultraschall Med 38(4):16-47. https://doi.org/10.1 055/a-0641-0076

11. Mueller S, Sandrin L (2010) Liver stiffness: a novel parameter for the diagnosis of liver disease. Hepat Med 2:49-67. https://doi.org/10.2147/hmer. s7394

12. Wang HW, Shi HN, Cheng J, Xie F, Luo YK, Tang J (2018) Real-time shear wave elastography (SWE) assessment of short- and long-term treatment outcome in Budd-Chiari syndrome: a pilot study. PLoS One 13(5):e0197550. https://doi.org/10.1371/journal.pone.0197550

13. (1994) Intraobserver and interobserver variations in liver biopsy interpretation in patients with chronic hepatitis C. The French METAVIR Cooperative Study Group. Hepatology 20(1 pt1):15-20

14. Mukund A, Pargewar SS, Desai SN, Rajesh S, Sarin SK (2017) Changes in liver congestion in patients with Budd-Chiari syndrome following endovascular interventions: assessment with transient elastography. J Vasc Interv Radiol 28(5):683-687. https://doi.org/10.1016/j.jvir.2016.11.091

15. Ferraioli G, Tinelli C, Dal Bello B et al (2012) Accuracy of real-time shear wave elastography for assessing liver fibrosis in chronic hepatitis $\mathrm{C}$ : a pilot study. Hepatology (Baltimore, Md) 56(6):2125-2133. https://doi.org/10.1002/ hep. 25936

16. Jiang T, Tian G, Zhao Q et al (2016) Diagnostic accuracy of 2D-shear wave elastography for liver fibrosis severity: a meta-analysis. PLoS One 11(6): e0157219. https://doi.org/10.1371/journal.pone.0157219

17. Dajti E, Ravaioli F, Colecchia A et al (2019) Liver and spleen stiffness measurements for assessment of portal hypertension severity in patients with Budd Chiari syndrome. Can J Gastroenterol Hepatol:1673197. https:/ doi.org/10.1155/2019/1673197

18. Samir AE, Manish D, Abhinav V et al (2015) Shear-Wave elastography for the estimation of liver Fibrosis in chronic liver Disease: Determining Accuracy and Ideal Site for Measurement Radiology 274(3):888-896. https://doi.org// $0.1148 /$ radiol.14140839

19. Foucher J, Chanteloup E, Vergniol J et al (2006) Diagnosis of cirrhosis by transient elastography (FibroScan): a prospective study. Gut 55(3):403-408. https://doi.org/10.1136/gut.2005.069153

20. Mancuso A, Armata M, Politi F et al (2020) Controversies in Budd-Chiari Syndrome Management: Potential Role of Liver Stiffness. Am J Gastroenterol 115:952-953. https://doi.org/10.14309/ajg.0000000000000586

21. Nakatsuka T, Soroida Y, Nakagawa $\mathrm{H}$ et al (2019) Utility of hepatic vein waveform and transient elastography in patients with Budd-Chiari syndrome who require angioplasty: Two case report. Medicine (Baltimore) 98(45):e17877. https://doi.org/10.1097/md.0000000000017877

\section{Publisher's Note}

Springer Nature remains neutral with regard to jurisdictional claims in published maps and institutional affiliations.

\section{Submit your manuscript to a SpringerOpen ${ }^{\circ}$ journal and benefit from:}

- Convenient online submission

- Rigorous peer review

- Open access: articles freely available online

- High visibility within the field

- Retaining the copyright to your article

Submit your next manuscript at $\boldsymbol{\nabla}$ springeropen.com 